\title{
Effects of a Custom-Made Pillow on Sleep and Blood Pressure Circadian Rhythm
}

\author{
Seiko Miyata and Akiko Noda*
}

Department of Biomedical Sciences, Chubu University, 1200 Matsumoto-cho Kasugai-shi, Aichi 487-8501, Japan

\begin{abstract}
Background: Sleep problems and the blood pressure (BP) circadian rhythm are closely related. A short duration of sleep is linked to a higher 24-h BP and nighttime BP in the younger generation. We studied the effect of a custom-made pillow on sleep and BP in young healthy adults.

Methods: This study included 17 young adults (12 males and five females; mean age, $22.2 \pm 3.2$ years). We evaluated satisfaction using each pillow, sleep indices, and 24-h BP when using conventional and custom-made pillows. Satisfaction using the pillow was evaluated using a visual analog scale (VAS). All of the measurements were made after one-month use of each of the two pillows. All of the subjects wore an accelerometry-based activity monitor and the actigraph data were assessed by the Sleep Sign Act software. We calculated sleep efficiency (total sleep time/time in bed $\times 100$ ). We evaluated nocturnal BP fall ([daytime BP - nighttime BP]/daytime BP×100) using 24-hour ambulatory BP monitoring.

Results: The VAS score when using the custom-made pillow was significantly greater than that when using the conventional pillow. Sleep efficiency increased after one-month use of the custom-made pillow in 11 subjects whose sleep efficiency was $<90 \%$ when using a conventional pillow. The nocturnal BP fall was significantly correlated with sleep efficiency.
\end{abstract}

Conclusion: Appropriate pillow use for one month could have a good effect on the BP circadian rhythm.

Keywords: 24-hour blood pressure monitoring; Actigraphy; Custom-made pillow; Dipper; Nondipper; Sleep efficiency

\section{Introduction}

Our contemporary 24-hour (h) society influences circadian rhythms and impairs the quality of life, resulting in tiredness, emotional instability, poor judgment, etc. These adverse effects are important factors related to sleep and psychiatric disorders. Moreover, short sleep duration and bad sleep quality are closely associated with lifestylerelated diseases. Various products for obtaining good-quality sleep have been developed with the increasing recognition of the importance of getting comfortable sleep $[1,2]$.

Sleep problems and the blood pressure (BP) circadian rhythm are closely related. A short duration of sleep is linked to a higher 24-h BP and nighttime BP in the younger generation [3]. Increased nighttime activity has been found to influence the daytime BP rhythm, causing a smaller reduction in nighttime BP [4]. Moreover, disturbance of the BP circadian rhythm is a risk factor for cardiovascular diseases such as cerebral infarction and hemorrhage [5]. Poor sleep quality, defined as Pittsburgh sleep quality index (PSQI) $\geq 6$, was significantly associated with resistance to treatment in hypertensive women, independent of cardiovascular and psychiatric confounders [6].

The purpose of study was to investigate the effects of one-month use of a custom-made pillow on sleep and BP in young adults.

\section{Methods}

\section{Study subjects}

This study included 17 young adults ( 12 males and five females; mean age, $22.2 \pm 3.2$ years). None of the subjects had a history or symptoms of cardiac, vascular, pulmonary, metabolic, or neurological disease, and none had taken medication before or during the study. We evaluated satisfaction using each pillow, sleep indices, and 24-h BP when using conventional and custom-made pillows. All of the measurements were made after one-month use of each of the two pillows. The order of pillow use was randomly allocated. The Chubu University ethics committee approved all of the procedures associated with the study. We obtained written informed consent from each participant after the nature of the procedures had been fully explained.

\section{Pillow fitting}

The custom-made pillow was $43 \times 63 \mathrm{~cm}$ in size and consisted of graining cotton, small- and large-sized beads, and a cotton pillowcase (TANAKA FUTON SERVICE CO., LTD, Ichinomiya, Japan). The pillow was fitted to each subject by three measurements of the head, neck and shoulder. Based on the differences in the shoulder-to-head length and the shoulder-to-neck length, 14 pockets inside the pillow were filled with cotton and beads and arranged to fit the subject's sleep position and to make the subject feel comfortable.

\section{Self-evaluation of satisfaction with pillow}

Satisfaction using the pillow was evaluated using a Visual Analog Scale (VAS). VAS data were recorded in millimeters with a range from 0 to 100 . The subjects were asked to mark the line between the two extremes reflecting their degree of satisfaction using the pillow

*Corresponding author: Akiko Noda, Department of Biomedical Sciences, Chubu University, 1200 Matsumoto-cho Kasugai-shi, Aichi 487-8501, Japan, Tel: 81-568-51-9906; Fax: 81-568-51-5370; E-mail: anoda@isc.chubu.ac.jp

Received September 08, 2014; Accepted October 13, 2014; Published October 17, 2014

Citation: Miyata S, Noda A (2014) Effects of a Custom-Made Pillow on Sleep and Blood Pressure Circadian Rhythm. J Hypertens 3: 183. doi:10.4172/2167-1095.1000183

Copyright: $\odot 2014$ Miyata S, et al. This is an open-access article distributed under the terms of the Creative Commons Attribution License, which permits unrestricted use, distribution, and reproduction in any medium, provided the original author and source are credited. 
Citation: Miyata S, Noda A (2014) Effects of a Custom-Made Pillow on Sleep and Blood Pressure Circadian Rhythm. J Hypertens 3: 183. doi:10.4172/2167-1095.1000183

Page 2 of 3

(ranging from "very bad" to "very good"). VAS is the most commonly used measure of pain in clinical settings [7].

\section{Sleep evaluation}

All of the subjects wore an accelerometry-based activity monitor (Lifecorder GS/Me, Suzuken Co., Nagoya, Japan) on their waist for one week. The actigraph data were assessed using the algorithm supplied by the SleepSign Act software (Kissei Comtec, Matsumoto, Japan) [8]. We evaluated sleep efficiency (total sleep time/time in bed $\times 100$ ). All of the subjects maintained one-week sleep logs, which were used to collect information regarding daily timing (i.e., bedtime and wakeup time) of sleep, and the time the actigraph was taken off.

\section{Blood pressure evaluation}

We measured 24-h ambulatory BP using a FB-270 oscillometric monitor (Fukuda Denshi, Tokyo, Japan). BP was continuously measured every 30 minutes overa 24 -h period. Artifactual readings were identified by trained technologists and omitted from analyses. A nocturnal BP fall was calculated as (daytime BP - nighttime BP)/ daytime $\mathrm{BP} \times 100$. The subjects with nocturnal $\mathrm{BP}$ fall of $<10 \%$ in systolic BP were classified as nondippers, and subjects with nocturnal BP fall of $>10 \%$ in systolic BP were classified as dippers [9].

\section{Statistical analysis}

All data are expressed as mean $\pm \mathrm{SD}$. We used paired $t$ test to compare satisfaction of pillow, sleep efficiency, night systolic BP (SBP), night diastolic BP (DBP), and BP fall between use of the conventional pillow and use of the custom-made pillow. The change in nocturnal BP fall ( $\Delta$ nocturnal BP fall), change in sleep efficiency $(\Delta$ sleep efficiency) and change in VAS ( $\triangle$ VAS) were calculated as values after one-month use of custom-made pillow - values after one-month use of conventional pillow. The correlations among $\Delta$ nocturnal BP fall, $\Delta$ sleep efficiency and $\Delta$ VAS were analyzed by Pearson's correlation analysis. All analyses were performed using the SPSS Software 20.0 package (IBM Corp., Armonk, NY, USA). P-values of $<0.05$ were considered statistically significant.

\section{Results}

The VAS score when using the custom-made pillow was significantly greater than that when using the conventional pillow (73 \pm 22 vs. $53 \pm 24, \mathrm{P}<0.05)$.

Sleep efficiency $<90 \%$ was observed in 13 subjects (76.4\%) when using the conventional pillow. Sleep efficiency increased after one month of using the custom-made pillow in 11 subjects $(84.6 \%, 11 / 13)$ whose sleep efficiency was $<90 \%$ when using the conventional pillow.

Seven subjects $(41.1 \%)$ were nondippers on 24-h BP monitoring when using the conventional pillow (Figure 1). Five of these subjects $(71.4 \%, 5 / 7)$ improved to dipper status with the use of the custommade pillow. The nocturnal BP fall when using the custom-made pillow was significantly greater than that when using the conventional pillow ( $14 \pm 4$ vs. $11 \pm 5 \%, \mathrm{n}=17, \mathrm{P}<0.05$ ). No significant differences were observed in 24-h, daytime and nighttime SBP or DBP when using the conventional or custom-made pillow (Table 1).

The $\Delta$ nocturnal BP fall was significantly correlated with $\Delta$ sleep efficiency $(\mathrm{r}=0.620, \mathrm{P}<0.05)$. However, there was no significant correlation between $\Delta$ nocturnal BP fall and $\Delta \mathrm{VAS}$ (Figure 2).

\section{Discussion}

In the present study, $84.6 \%$ of the subjects with sleep efficiency $<90 \%$ exhibited an increase in sleep efficiency upon using the custommade pillow. On 24-h BP monitoring, 71.4\% of the subjects who were nondippers improved to dipper status after using the custom-made pillow. The change in nocturnal BP fall was significantly correlated with the change in sleep efficiency. These results suggest that the use of an appropriate pillow can improve BP circadian rhythm.

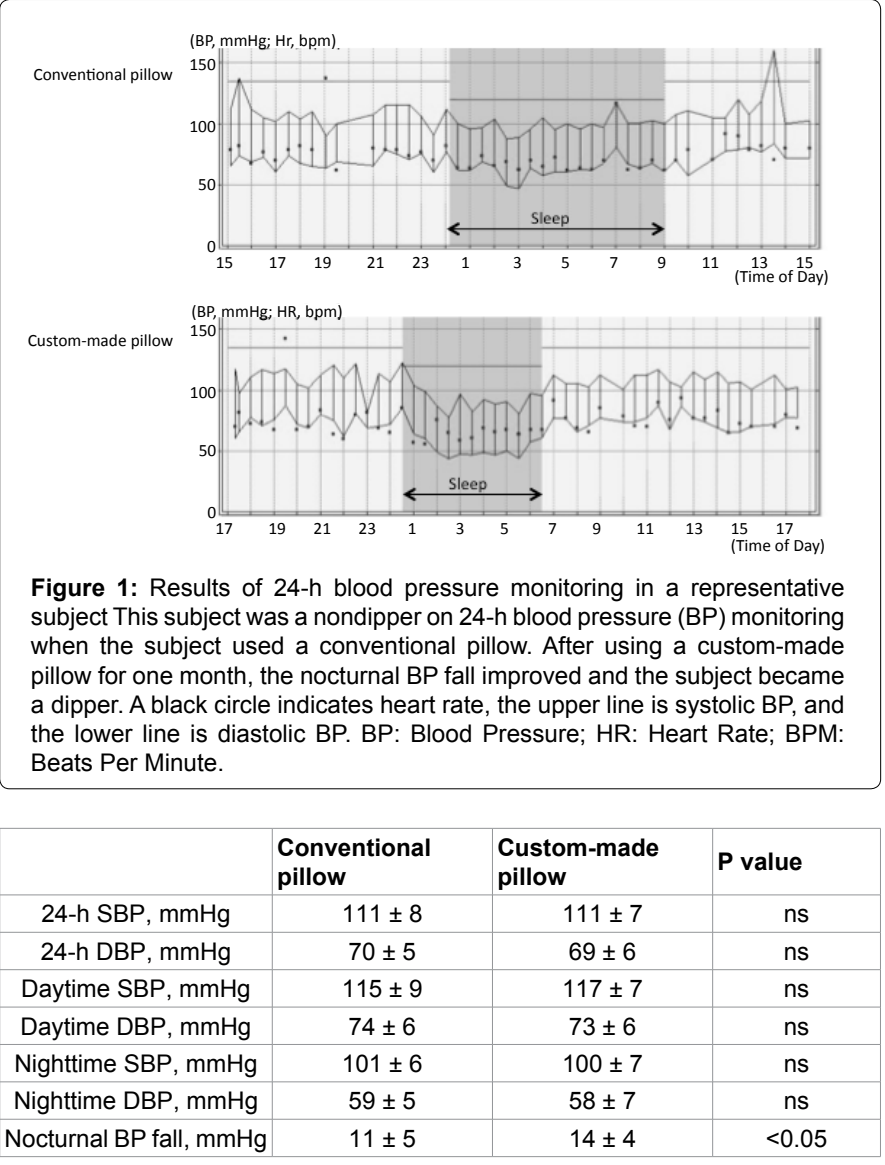

Table 1: Comparison of 24-h blood pressure monitoring between conventional and custom-made pillows, SBP: Systolic Blood Pressure; DBP: Diastolic Blood Pressure; NS: Not Significant.
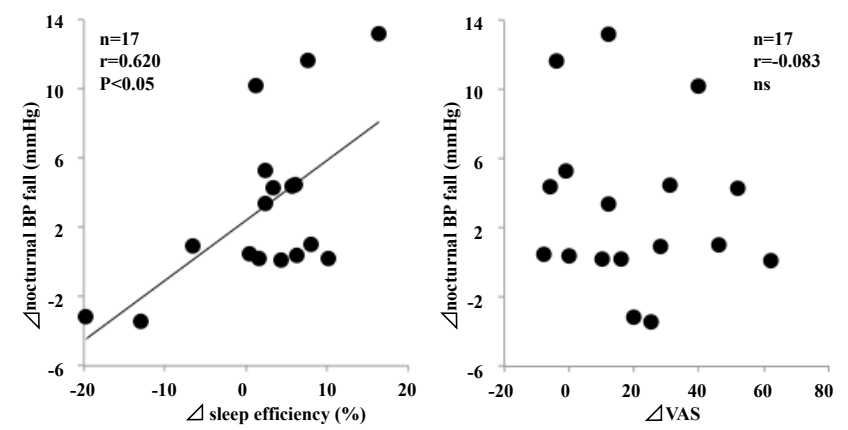

Figure 2: The relationships among changes in nocturnal blood pressure fall, sleep efficiency and visual analogue scale. $\Delta$ nocturnal BP fall, $\Delta$ sleep efficiency and $\triangle$ VAS were calculated as values after one-month use of custom-made pillow - values after one-month use of conventional pillow. BP: Blood Pressure; VAS: Visual Analogue Scale. 
Citation: Miyata S, Noda A (2014) Effects of a Custom-Made Pillow on Sleep and Blood Pressure Circadian Rhythm. J Hypertens 3: 183. doi:10.4172/2167-1095.1000183

Page 3 of 3

The degree of satisfaction using the custom-made pillow was significantly greater than that using the conventional pillow, and $84.6 \%$ of subjects with sleep efficiency $<90 \%$ exhibited an increase in sleep efficiency upon using the custom-made pillow. Many people appear to make poor pillow choices because low pillow comfort and waking due to cervico-thoracic symptoms have been reported [1]. A pillow fitted to the cervical-spine shape of the subject relieved headache and waking due to cervico-thoracic symptoms and improved subjective sleep quality [2]. These studies support our data that subjective satisfaction significantly increased when the subjects used the custom-made pillow.

Although $41.1 \%$ of the subjects were nondippers on 24 -h BP monitoring when using the conventional pillow, $71.4 \%$ of these subjects improved to dipper status when using the custom-made pillow. The subjects who reached a better circadian BP rhythm showed increased sleep efficiency. The nocturnal BP fall was significantly correlated with sleep efficiency in the present study. Day-to-day BP change is associated with sleep efficiency [10]. A short duration of sleep is also related to increases in systolic BP and diastolic BP and decrease in the sleep/wake BP ratio [3]. These findings indicate that a custom-made pillow may increase sleep efficiency, resulting in normalization of the BP circadian rhythm.

Eleven of 13 subjects with sleep efficiency $<90 \%$ when using the conventional pillow exhibited an increase in sleep efficiency upon using the custom-made pillow. Previous studies evaluated only the subjective sleep satisfaction, and did not fully perform objective sleep evaluation [1,2]. Subjective evaluation is a convenient and less stressful tool in a large-scale study. However, its reliability is inferior to that of an objective evaluation. In addition to a physical examination, an assessment of pillow fit could be important in individualswith sleep problems.

\section{Conclusion}

In the present study, one-month use of a custom-made pillow improved sleep efficiency and the 24-h BP circadian rhythm in more than half of the subjects with abnormal values when using a conventional pillow, suggesting the potential benefit of using a custommade pillow on the 24-h BP circadian rhythm.

\section{Acknowledgements}

This study was funded by research grants from the Ministry of Education Culture, Sports, Science, Technology of Japan, Aichi Science \& Technology Foundation of Japan and TANAKA FUTON SERVICE CO., LTD.

\section{References}

1. Liu SF, Lee YL, Liang JC (2011) Shape design of an optimal comfortable pillow based on the analytic hierarchy process method. J Chiropr Med 10: 229-239.

2. Persson $L$ (2012) Neck pain and pillows-A blinded study of the effect of pillows on non-specific neck pain, headache and sleep. Adv Physiother 8: 122-7.

3. Mezick EJ, Hall M, Matthews KA (2012) Sleep duration and ambulatory blood pressure in black and white adolescents. Hypertension 59: 747-752.

4. Cavelaars M, Tulen JH, van Bemmel JH, van den Meiracker AH (2004) Physical activity, dipping and haemodynamics. J Hypertens 22: 2303-2309.

5. Metoki H, Ohkubo T, Imai $Y$ (2010) Diurnal blood pressure variation and cardiovascular prognosis in a community-based study of Ohasama, Japan. Hypertens Res 33: 652-656.

6. Bruno RM, Palagini L, Gemignani A, Virdis A, Di Giulio A, et al. (2013) Poor sleep quality and resistant hypertension. Sleep Med 14: 1157-1163.

7. Scott J, Huskisson EC (1976) Graphic representation of pain. Pain 2: 175-184

8. Enomoto M, Endo T, Suenaga K, Miura N, Nakano Y, et al. (2009) Newly developed waist actigraphy and its sleep/wake scoring algorithm. Sleep Biological Rhythm 7: 17-22.

9. Kario K, Pickering TG, Matsuo T, Hoshide S, Schwartz JE, et al. (2001) Stroke prognosis and abnormal nocturnal blood pressure falls in older hypertensives. Hypertension 38: 852-857.

10. Hinderliter AL, Routledge FS, Blumenthal JA, Koch G, Hussey MA, et al. (2013) Reproducibility of blood pressure dipping: relation to day-to-day variability in sleep quality. J Am Soc Hypertens 7: 432-439. 